\title{
New and Emerging Systemic Treatments for Atopic Dermatitis
}

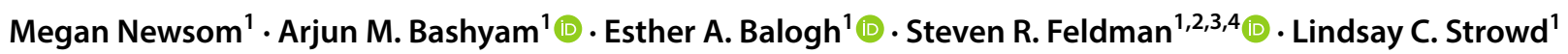

Published online: 9 June 2020

(c) Springer Nature Switzerland AG 2020

\begin{abstract}
Atopic dermatitis (AD) is a prevalent inflammatory skin condition that, depending on its severity, can cause enormous morbidity. Corticosteroids and systemic immunosuppression, traditionally standard of care for difficult-to-treat disease, have many undesirable side effects. The desire for targeted treatments along with an improved understanding of the pathophysiology of AD has spurred the development of novel treatments. In this article, we review promising new treatments and discuss how their targets-IL-13, IL-31, OX40 (CD134), and the Janus kinase family of proteins-participate in the pathogenesis of AD. We review the published phase II and III data for dupilumab, tralokinumab, lebrikizumab, nemolizumab, anti-OX40 antibody, baricitinib, abrocitinib, and upadacitinib. The introduction of new agents may offer new options, but it remains to be seen how narrow-acting agents, like single interleukin inhibitors, will compare in safety and efficacy to broad-acting agents such as JAK inhibitors.
\end{abstract}

\section{Key Points}

Our better understanding of the pathophysiology of AD has resulted in an explosion of research into new immunotherapies for this patient population.

Multiple new agents targeting IL-13, IL-31, OX40 (CD134), and Janus kinase proteins may be effective for AD.

Megan Newsom

mnewsom@wakehealth.edu

1 Department of Dermatology, Center for Dermatology Research, Medical Center Boulevard, Wake Forest School of Medicine, Winston-Salem, North Carolina, 27157-1071, USA

2 Department of Pathology, Wake Forest School of Medicine, Winston-Salem, NC, USA

3 Department of Social Sciences and Health Policy, Wake Forest School of Medicine, Winston-Salem, NC, USA

4 Department of Dermatology, University of Southern Denmark, Odense, Denmark

\section{Introduction}

Atopic dermatitis (AD) is a common inflammatory skin disease characterized by pruritus and skin barrier dysfunction [1-3]. Current mainstay treatments include topical moisturizers, topical corticosteroids, topical calcineurin inhibitors, phototherapy, and systemic immunotherapies [4]. Moderateto-severe $\mathrm{AD}$ is often refractory to first-line topical treatments; while systemic immunosuppressants are efficacious, they have significant adverse effects [4].

The shortcomings of mainstay treatments prompted the development of targeted topical and systemic immunotherapies involving pathways directly responsible for AD. The US Food and Drug Administration (FDA) approved a topical phosphodiesterase-4 $\left(\mathrm{PDE}_{4}\right)$ inhibitor, crisaborole, in 2016 for mild-to-moderate AD and a monoclonal antibody, dupilumab, in 2017 for moderate to severe AD [5]. While the efficacy of dupilumab is considerable, the clinical success 
of crisaborole is less impressive. Additional new treatments are desirable, as $\mathrm{AD}$ is a heterogeneous disease with several immunologic phenotypes [3]. The purpose of this review is to discuss the mechanisms, safety, and efficacy of the new and upcoming systemic immunologic treatments for AD.

\section{Immunology of Atopic Dermatitis}

Atopic dermatitis is a disease without a single identifiable pathophysiological cause $[3,6]$. Several subtypes of AD exist, including extrinsic, intrinsic, pediatric-onset, and hand and foot $[3,7,8]$. These subtypes have different inciting factors and molecular compositions [7]. For example, IgE levels are only elevated in about $20-50 \%$ of patients, and loss-of-function mutations in the filaggrin $(F L G)$ gene are only identified in a small subset of AD patients of European ancestry [1, 4, 9]. However, all subtypes of AD are characterized by a cycle of $\mathrm{T}$ cell mediated skin inflammation and disruption of the skin barrier $[8,10]$.
Effector immune cells are recruited to sites of skin damage when injured keratinocytes release pro-inflammatory signals. In the acute phase, type 2 helper $\mathrm{T}$ cells $\left(\mathrm{T}_{\mathrm{H}} 2\right)$, type 17 helper $\mathrm{T}$ cells $\left(\mathrm{T}_{\mathrm{H}} 17\right)$, and type 22 helper $\mathrm{T}$ cells $\left(\mathrm{T}_{\mathrm{H}} 22\right)$ predominate. Increased type 1 helper $\mathrm{T}$ cell $\left(\mathrm{T}_{\mathrm{H}} 1\right)$ activation along with $\mathrm{T}_{\mathrm{H}} 2$ and $\mathrm{T}_{\mathrm{H}} 22$ inflammation characterizes the chronic phase of the disease [10]. Cytokines, such as thymic stromal lymphopoietin (TSLP), interleukin (IL)-25, and IL-33 promote the maturation of skin resident $\mathrm{T}_{\mathrm{H}} 2$ and group 2 innate lymphoid cells (ILC2s) [1, 4]. ILC2s are tissue-resident lymphocytes that do not derive from either the $\mathrm{T}$ cell or $\mathrm{B}$ cell lineage. Along with $\mathrm{T}_{\mathrm{H}} 2$ cells, ILC2s produce a large amount of the pro-inflammatory cytokine IL-13 [1, 11].

When IL-4 or IL-13 binds to either type (I or II) of the IL-4 receptor complex, an associated Janus kinase (JAK) protein-JAK1, JAK2, JAK3, or tyrosine kinase 2 (TYK2)is phosphorylated and activated (Fig. 1) [11]. Activation of JAK proteins leads to a phosphorylation cascade, which ultimately activates the transcription factors signal transducer

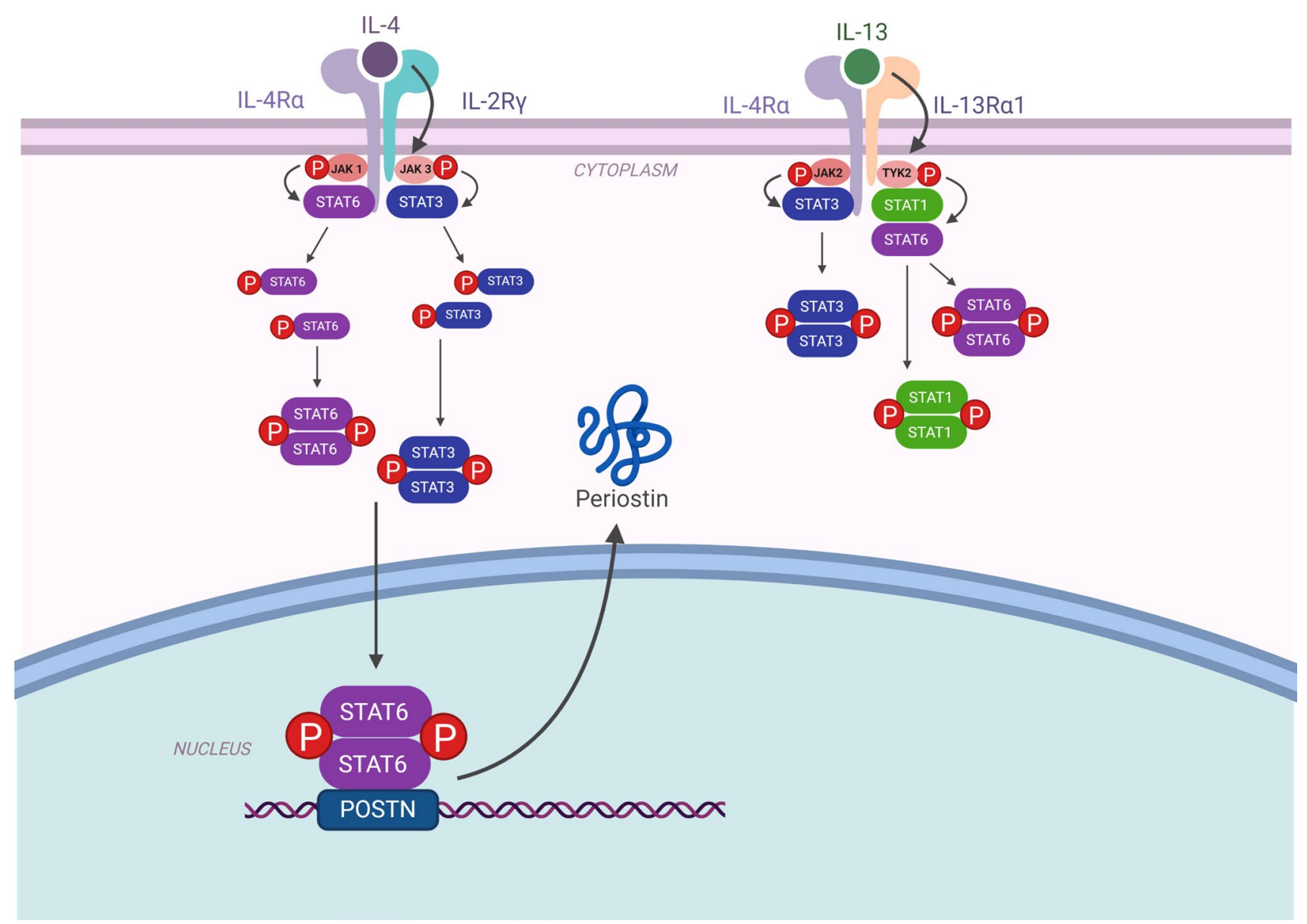

Fig. 1 IL-4 and IL-13 signaling pathways via JAK-STAT signaling cascade. Created with biorender.com. IL interleukin, JAK Janus kinase, TYK2 tyrosine kinase 2, STAT signal transducer and activator of transcription 
and activator of transcription 6 (STAT6) and signal transducer and activator of transcription 3 (STAT3) [11].

Many proteins essential for skin-barrier function-including filaggrin, loricrin, involucrin, and ceramides-are downregulated or inhibited in this way through the effect of IL-4 and IL-13 on gene expression [4]. Additionally, activation of STAT6 results in increased gene expression of periostin, a pro-inflammatory extracellular matrix protein, trophic to keratinocytes that stimulates them to produce TSLP [1]. $\mathrm{T}_{\mathrm{H}} 2$ cells also express IL-31, which acts on keratinocytes to potentiate the release IL-24. This, in turn, leads to decreased FLG production and resultant skin barrier breakdown $[1,4]$.

\section{Agents Targeting Interleukin-13 or Its Receptors}

IL-13 is a suitable therapeutic target in the treatment of $\mathrm{AD}$, as increased levels of IL-13 correlate well with disease severity $[1,11,12]$. Preventing IL-13 signaling is the basis for three monoclonal antibody treatments for refractory $\mathrm{AD}$-dupilumab, tralokinumab, and lebrikizumab.

\subsection{Dupilumab}

Dupilumab binds to IL-4R $\alpha$, a component of both the IL-4 and IL-13 receptors essential for pro-inflammatory signal transduction [1, 11]. Additionally, by inhibiting activation of the IL-4R $\alpha$ on sensory nerves, the sensation of pruritus is decreased [1]. In comparison with systemic immunosuppressants like methotrexate and cyclosporine, dupilumab is dosed more conveniently (two initial injections and then one injection every 2 weeks) and provides more targeted immunomodulation.

Several clinical trials support dupilumab's clinical success in treating moderate-to-severe AD (Table 1). In the phase III SOLO-1 randomized controlled trial (RCT), an investigator global assessment (IGA) score of 0 or 1 plus $\geq 2$-point improvement from baseline was considered success. By week 16, a larger percentage of patients receiving dupilumab achieved success compared with the group receiving placebo (Table 1) [13]. Additionally, a higher proportion of patients receiving dupilumab achieved Eczema Area and Severity Index (EASI)-75 compared with the group receiving placebo. These results were replicated in the phase III SOLO-2 trial and the phase III LIBERTY AD CAFE trial (Table 1) [13, 14].

In the 76-week open-label long-term extension study, $88.4 \%$ of subjects achieved an EASI-75 compared with the baseline of the parent study and $58.0 \%$ of subjects achieved a 2-point or greater improvement in IGA score compared with baseline [15]. In a phase III RCT in adolescents, by week 16, a larger percentage of the group receiving dupilumab (either every 2 weeks [41.5\%] or every 4 weeks [38.1\%]) achieved an EASI-75 when compared with the group receiving placebo $(8.2 \% ; p<0.001$ for both) [16]. Additionally, by week 16 , a larger percentage of the group receiving dupilumab (either every 2 weeks [24.4\%] or every 4 weeks [17.9\%]) achieved an IGA of 0 or 1 compared with the group receiving placebo (2.4\%; $p<0.001$ for both) [16].

Dupilumab had an acceptable safety profile in clinical trials, which has borne out in clinical practice [17]. Idiopathic and allergic conjunctivitis can occur with dupilumab use, although this side effect is rarely treatment limiting $[13,18,19]$. In the long-term open-label extension study of dupilumab, $18 \%$ of the group receiving $2 \mathrm{mg}$ per $\mathrm{kg}$ of dupilumab and $16 \%$ of the group receiving $4 \mathrm{mg}$ per $\mathrm{kg}$ of dupilumab reported conjunctivitis [20]. Conjunctivitis is less likely to occur with dupilumab treatment in other $\mathrm{T}_{\mathrm{H}} 2$-driven diseases such as asthma [21]. Additionally, there are several case reports of the development of alopecia areata (AA) after starting dupilumab [22-24]. However, patients with $\mathrm{AD}$ have higher rates of AA, and clinical trials found no increased risk in the groups receiving dupilumab compared with placebo [25]. Mouse models of IL-4R $\alpha$ deletions indicate increased vulnerability to helminthic infections. This is attributed to the necessity of this subunit in dendritic cell maturation. However, an increase in parasitic infections has not been reported in humans using dupilumab [11].

Understanding the clinical effectiveness of dupilumab will impact the reception of the novel agents discussed in later sections. While the trial data presented here may suggest that dupilumab is only modestly effective in moderateto-severe $\mathrm{AD}$, this underestimates dupilumab's ability to achieve clinically meaningful improvement. The primary outcome measures used in clinical trials (e.g., IGA 0/1 or EASI-75) are investigator-reported measures of disease clearance. However, investigator-reported outcomes do not correlate strongly with patient-reported outcomes, which are key to patients' quality of life - the goal of clinical treatment [26]. Also, while investigator-reported measures of lesion clearance are useful in clinical trials for distinguishing drug from placebo, there is evidence that they underestimate the percentage of patients who have clinically meaningful improvement [27]. This is supported by the results from studies evaluating dupilumab in the real-world setting [17, $28,29]$. Additionally, in clinical practice, when patients have only a partial response to systemic treatment, topical treatment can be added to achieve more complete clearing.

\subsection{Tralokinumab}

Tralokinumab is a humanized monoclonal $\mathrm{IgG}_{4}$ antibody (MAB) that neutralizes IL-13 [12]. In a phase IIb RCT, by week 12, there was a larger mean decrease from baseline in 


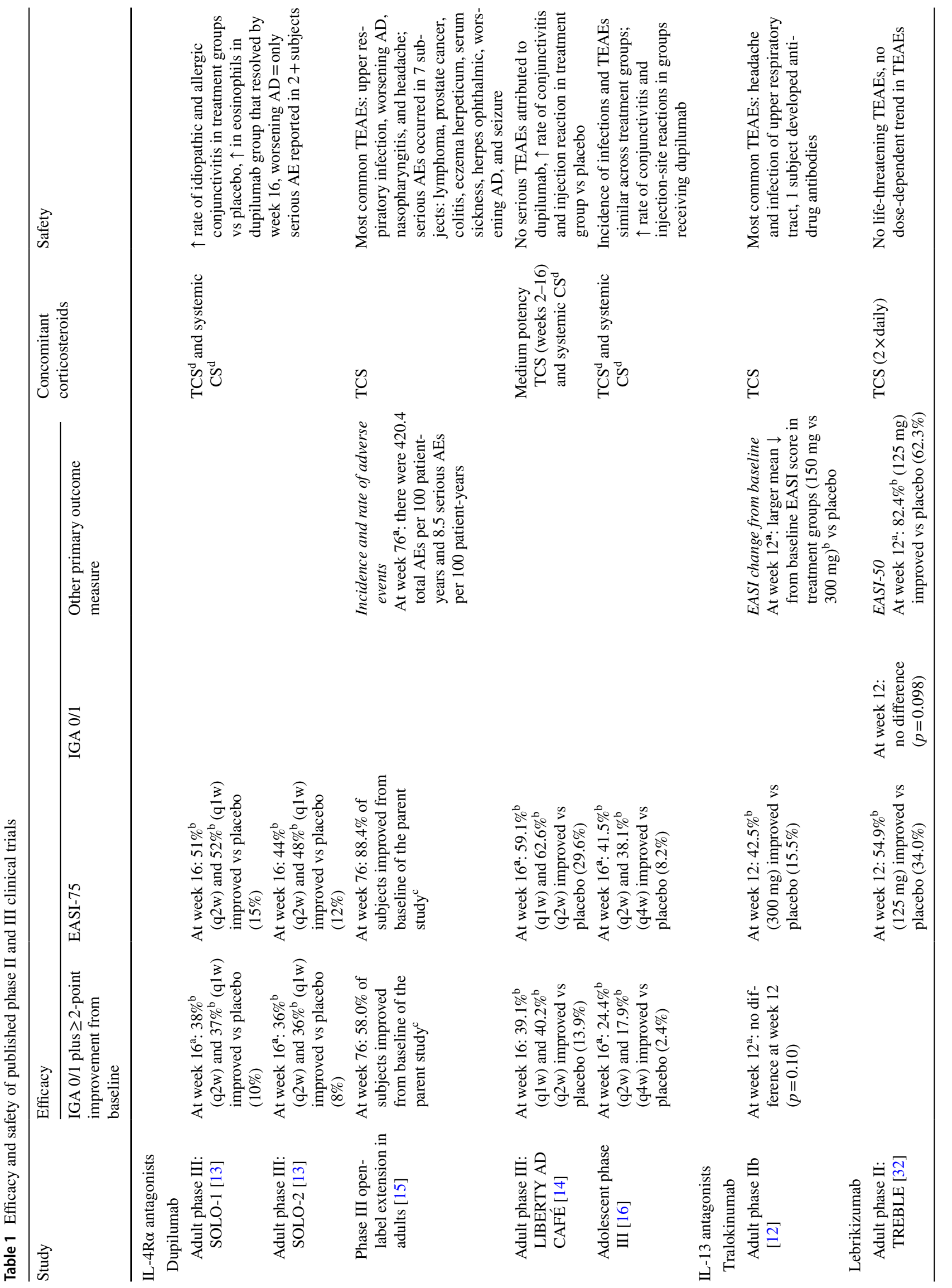




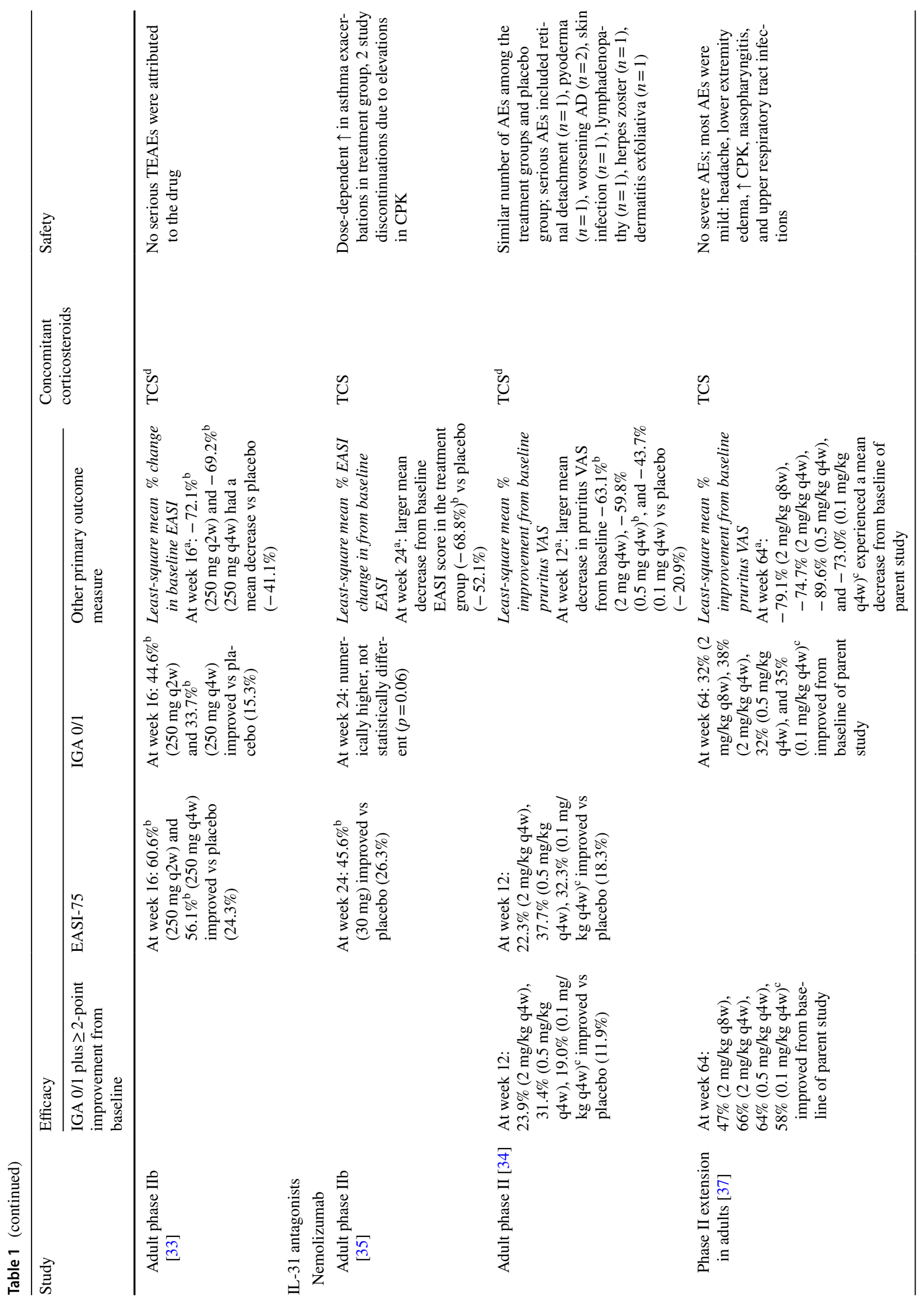




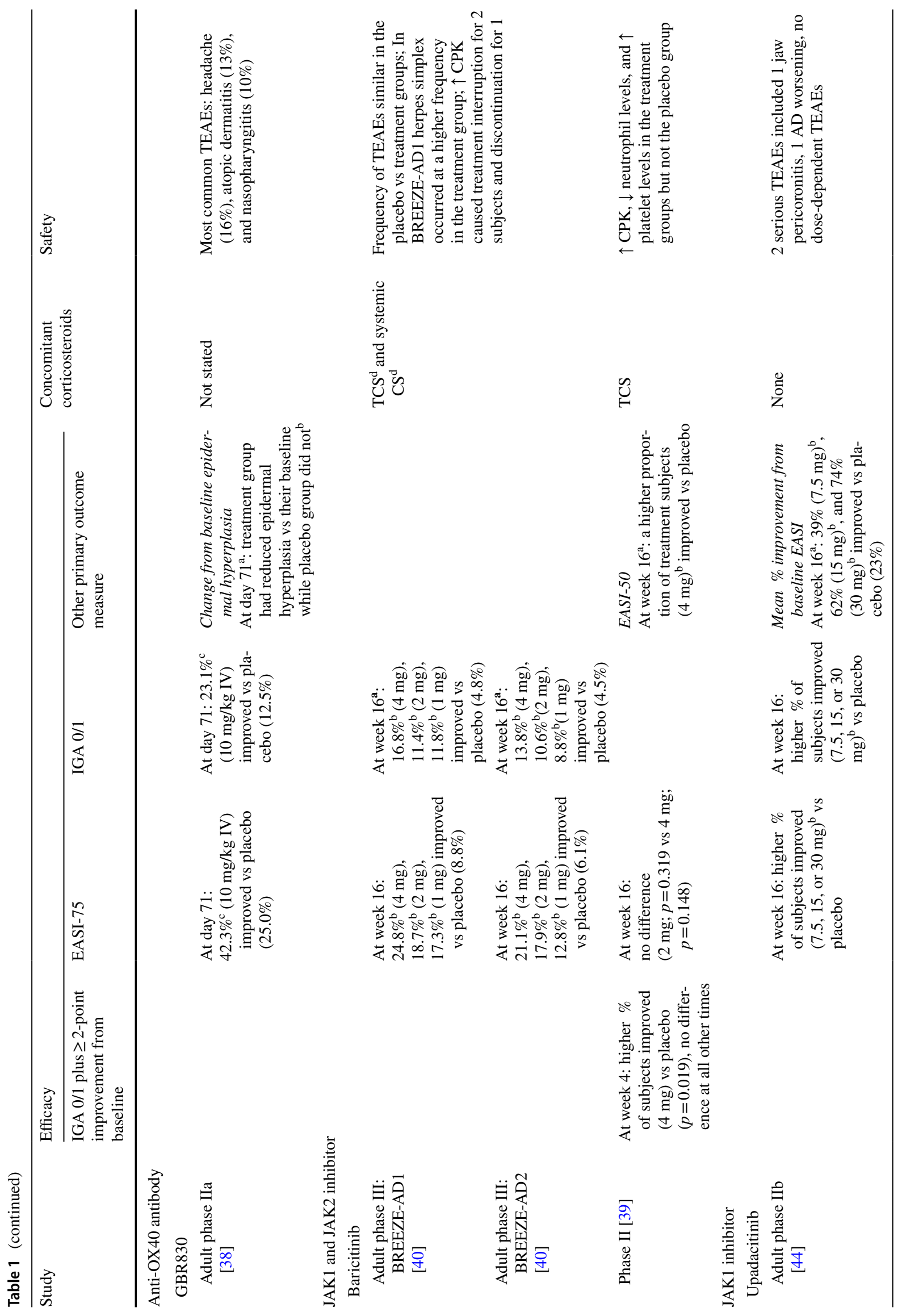




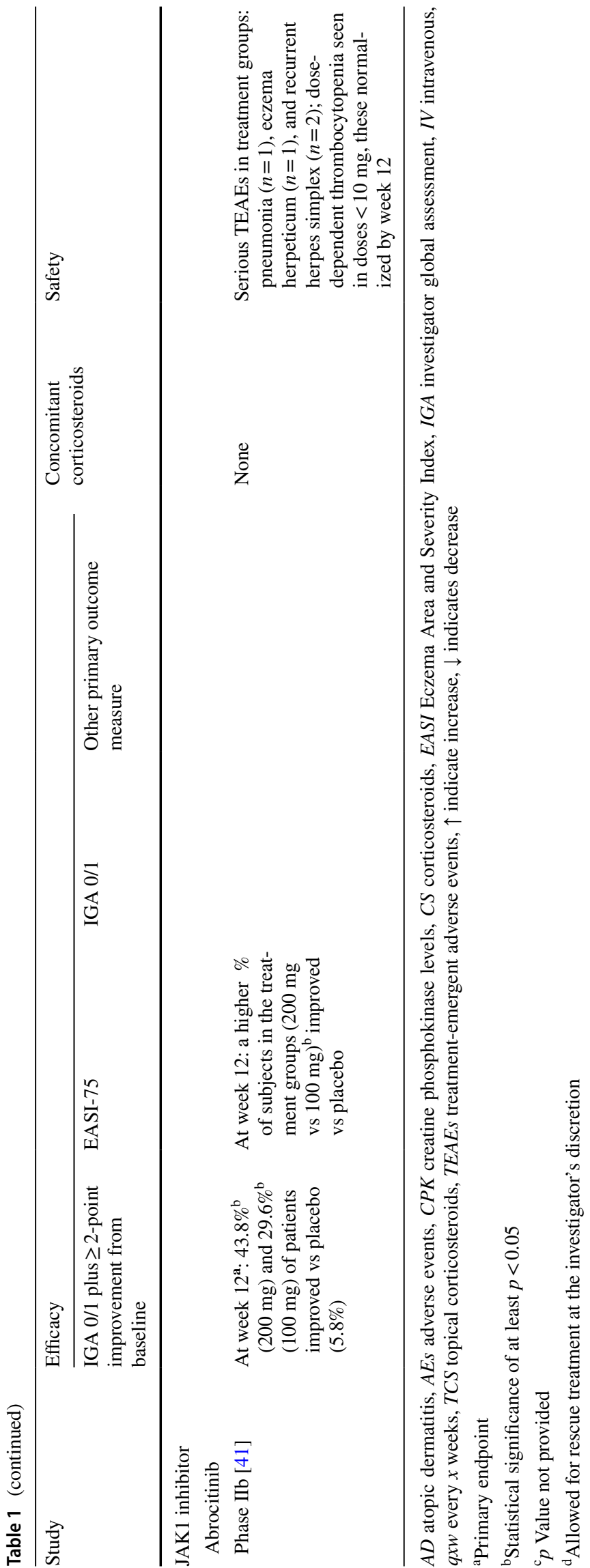

EASI score in the groups receiving tralokinumab $(150 \mathrm{mg}$ vs $300 \mathrm{mg}$ ) compared with placebo ( $p=0.03$ and $p=0.01$, respectively) [12]. Additionally, by week 12, a higher proportion of subjects achieved an EASI-75 in the group receiving $300 \mathrm{mg}$ of tralokinumab (42.5\%) compared with placebo $(15.5 \% ; p=0.003)$. However, there was no difference in the percentage of subjects achieving an IGA of 0 or 1 at 12 weeks in the pooled group of subjects receiving tralokinumab $(p=0.10)$. Recently, Leo Pharma announced positive preliminary results from the three phase III ECZema TRAlokinumab (ECZTRA 1-3) trials, although this data is not yet publicly available [30]. In a phase I study evaluating the safety of tralokinumab, headache and somnolence occurred in the treatment group but not in the placebo group [31]. In the phase IIb trial, the most common treatmentemergent adverse events (TEAEs) reported were headache and infection of the upper respiratory tract. Only one participant (of 153) developed a positive titer for anti-drug antibodies [12].

\subsection{Lebrikizumab}

Lebrikizumab is a MAB that binds IL-13, inhibiting the dimerization of IL-13R $\alpha 1$ and IL-4R $\alpha$ [32]. One ongoing phase III clinical trial is evaluating lebrikizumab in adults with AD (Table 2). In the phase II TREBLE trial, at 12 weeks, a higher proportion of subjects in the group receiving lebrikizumab $125 \mathrm{mg}$ every 4 weeks achieved an EASI-50 (82.4\%) compared with the group receiving placebo (62.3\%; $p=0.026)$ [32]. Additionally, at 12 weeks, a higher proportion of subjects in the group receiving lebrikizumab $125 \mathrm{mg}$ every 4 weeks achieved an EASI-75 (54.9\%) compared with the group receiving placebo (34.0\%; $p=0.036)$. There was no significant difference in the percentage of subjects achieving an IGA of 0 or 1 between the group receiving lebrikizumab $125 \mathrm{mg}$ every 4 weeks (33.3\%) and the group receiving placebo $(18.9 \% ; p=0.098)$. A second phase IIb trial in adults reported similar efficacy results (Table 1) [33]. In the TREBLE study, there were no life-threatening adverse events and no adverse events showed a dose-dependent trend [32].

\section{Agent Targeting Interleukin 31}

\subsection{Nemolizumab}

Nemolizumab (CIM331) is a MAB that binds the IL-31 receptor $\alpha$ component. This prevents IL-31 from acting on neurons, which inhibits the potentiation of the sensation of pruritus [34-36]. Several phase III clinical trials are ongoing for nemolizumab in AD patients (Table 2). In a 12-week phase II RCT with a 64-week extension, by 12 weeks there 
was a mean decrease in pruritus visual analog scale (VAS) from baseline in the group receiving nemolizumab $2.0 \mathrm{mg}$ every 4 weeks $(-63.1 \%)$ compared with placebo $(-20.9 \%$; $p<0.001$ ) [34]. By 64 weeks, improvement in pruritus VAS compared with baseline was sustained for subjects receiving $0.1 \mathrm{mg} / \mathrm{kg}$ every 4 weeks, $0.5 \mathrm{mg} / \mathrm{kg}$ every 4 weeks, $2.0 \mathrm{mg} /$ $\mathrm{kg}$ every 4 weeks, and $2.0 \mathrm{mg} / \mathrm{kg}$ every 8 weeks [37]. By 64 weeks, $68 \%, 68 \%$, and $66 \%$ of subjects receiving nemolizumab $0.1,0.5$, and $2.0 \mathrm{mg} / \mathrm{kg}$ every 4 weeks, respectively, achieved an EASI-75 and 74\% of subjects receiving $2.0 \mathrm{mg} /$ $\mathrm{kg}$ every 8 weeks achieved an EASI-75 [37]. In a phase IIb RCT, by week 24 , a higher proportion of subjects achieved an IGA of 0 or 1 in the group receiving nemolizumab $30 \mathrm{mg}$ (36.8\%) compared with the group receiving placebo $(21.1 \%$; $p=0.06$ ). By week 24 , a larger percentage of subjects in the group receiving nemolizumab $30 \mathrm{mg}$ achieved an EASI-75 $(45.6 \%)$ compared with the placebo group $(26.3 \% ; p=0.034)$ [35].

In the phase I trial, infections were the most commonly reported TEAE. Nasopharyngitis (3 of 27 subjects) and herpes simplex ( 2 of 27 subjects) were reported in the treatment group but not the placebo group. There were no dose-dependent adverse events [36]. In the long-term extension of a 12-week phase II trial, no severe adverse events occurred for up to 64 weeks after treatment with nemolizumab. Most adverse events were mild and included headache, lower extremity edema, increased creatine phosphokinase levels (CPK), nasopharyngitis, and upper respiratory tract infections [37]. A phase IIb clinical trial reported a dose-dependent increase in mild asthma exacerbations in subjects treated with nemolizumab. Two subjects discontinued the study due to elevations in creatine kinase levels [35].

\section{Agent Targeting OX40 (CD134)}

\subsection{Anti-0X40 Antibody}

Anti-OX40 antibody (also called GBR 830) is a humanized monoclonal $\mathrm{IgG}_{1}$ antibody targeting the costimulatory molecule OX40 (CD134) [38]. OX40 is expressed on activated antigen presenting cells and endothelium and is essential for T-cell expansion [38]. A phase IIb clinical trial is currently recruiting (Table 2). In the published phase IIa clinical trial conducted in adults, the primary study endpoints included incidence and characterization of adverse events, change in epidermal hyperplasia compared with baseline, and mRNA expression signatures from skin biopsy [38]. The treatment group had reduced epidermal hyperplasia (compared with their baseline) at 29 days $(p<0.01)$ and 71 days $(p<0.001)$ while the placebo group did not. IL-31, CCL11, CCL17, and thymic stromal lymphopoietin (TSLP) levels were all decreased in the treatment group compared with baseline by 71 days $(p<0.001)$. IL-4, IL-13, IL-17a, and IL-22 levels were not altered after treatment with GBR830. By day 71, in an intention-to-treat analysis, there was a larger proportion of subjects in the group receiving $10 \mathrm{mg} / \mathrm{kg}$ of IV GBR 830 (42.3\%) that achieved

Table 2 Ongoing clinical trials (as of April 10, 2020)

\begin{tabular}{llll}
\hline Drug & Clinical trial $^{\text {a }}$ & Phase & Status \\
\hline Lebrikizumab & NCT04146363 & III & Suspended (due to COVID-19) \\
Nemolizumab & NCT03989206 & III & Recruiting \\
& NCT03985943 & Recruiting & Recruiting \\
& NCT03989349 & III & Recruiting \\
Anti-OX40 antibody & NCT03568162 & IIb & Active, not recruiting \\
Baricitinib & NCT03435081 (BREEZE-AD5) & III & Completed \\
& NCT03733301 (BREEZE-AD7) & Enrolling by invitation \\
& NCT03559270 (BREEZE-AD6) & III & Active, not recruiting \\
NCT03334435 & III & Active, not recruiting \\
Abrocitinib & NCT03428100 & III & Recruiting \\
NCT03952559 & III & Recruiting \\
& NCT03422822 & III & Recruiting \\
& NCT03607422 & III & Recruiting \\
& NCT03569293 & III & Recruiting \\
& NCT03738397 & III & Recruiting
\end{tabular}

${ }^{a}$ ClinicalTrials.gov identifier (www.clinicaltrials.gov) 
an EASI-75 compared with the group receiving placebo $(25.0 \%$; $p$ value was not reported). By day 71 , there was a larger proportion of subjects in the group receiving $10 \mathrm{mg} /$ $\mathrm{kg}$ of IV GBR $830(23.1 \%)$ that achieved an IGA score of 0 or 1 compared with the group receiving placebo $(12.5 \%$; $p$ value was not reported). The most common TEAEs were headache (16\%), AD (13\%), and nasopharyngitis (10\%). Adverse events of moderate severity included one subject with facial edema in the placebo group, one subject with a dental abscess, and one subject with worsening AD in the treatment group [38].

\section{Agents Targeting the Janus Kinase Family of Proteins}

Several small-molecule JAK inhibitors are being actively investigated in the treatment of moderate to severe $\mathrm{AD}$, including baricitinib, abrocitinib, and upadacitinib. The JAK proteins are intracellular and, when activated, activate STAT proteins to dimerize and translocate to the cell nucleus to increase gene expression of inflammatory mediators [8]. Some of the following agents are selective for particular JAK proteins while others inhibit the whole family.

\subsection{Baricitinib}

Baricitinib is an oral, small-molecule, selective inhibitor of JAK1 and JAK2 [39]. There are ongoing phase III clinical trials (Table 2). In two phase III clinical trials, BREEZE-AD1 and BREEZE-AD2, by 16 weeks, a higher proportion of subjects in the treatment groups ( $1 \mathrm{mg}, 2 \mathrm{mg}$, and $4 \mathrm{mg}$ ) achieved an IGA of 0 or 1, a $\geq 2$-point improvement, and EASI-75 compared with the group receiving placebo (Table 1) [40]. In a phase II clinical trial in adults with $\mathrm{AD}$, by week 16 , a higher percentage of subjects receiving baricitinib $4 \mathrm{mg}$ with a topical corticosteroid (TCS) achieved an EASI-50 than subjects given placebo with TCS $(p=0.027)$ [39]. In contrast, by week 16, there was no difference in the proportion of subjects achieving EASI-75 in the group receiving baricitinib ( $2 \mathrm{mg}$ $[n=20] ; 4 \mathrm{mg}[n=30])$ and TCS compared with the group receiving placebo and TCS $(n=34 ; p=0.319$ and $p=0.148$, respectively). At 4 weeks, a higher proportion of subjects achieved IGA of 0 or 1 after receiving $4 \mathrm{mg}$ of drug and TCS compared with placebo $(p=0.019)$. At all other time points, there was no difference in the proportion of treatment subjects achieving IGA of 0 or 1 compared with placebo. No lifethreatening adverse events were reported in this study. One serious TEAE was reported (a benign colonic polyp) in one subject receiving baricitinib $4 \mathrm{mg}$ plus TCS. Several adverse events present in the treatment groups but not the placebo group include increased CPK levels, decreased neutrophil levels, and increased platelet levels. In the BREEZE-AD1 and 2 studies, the frequency of TEAEs was similar among the placebo and the treatment groups. In BREEZE-AD1, there was an increased rate of herpes simplex infections in the treatment groups compared with the placebo group but this was not seen in BREEZE-AD2. Elevations in CPK caused treatment suspension in two subjects receiving baricitinib and discontinuation in one subject.

\subsection{Abrocitinib}

Abrocitinib (PF-04965842) is an oral, small-molecule, selective inhibitor of JAK1 [41]. The recently released data from the phase III trial evaluating abrocitinib monotherapy in subjects 12 years and older, JADE COMPARE, is promising. By 12 weeks, a significantly higher proportion of subjects in the treatment groups $(100 \mathrm{mg}$ or $200 \mathrm{mg}$ daily) achieved an IGA of 0 or 1 and a $\geq 2$-point improvement than the group receiving placebo. The proportion of subjects achieving an EASI-75 was also significantly higher in the treatment groups than the placebo group at 12 weeks [42]. In a phase IIb trial evaluating abrocitinib in moderate-to-severe $\mathrm{AD}$ by week 12 , a higher proportion of subjects receiving $200 \mathrm{mg}$ of drug and $100 \mathrm{mg}$ of drug (43.8\% and $29.6 \%$ ) had an IGA of 0 or 1 plus a $\geq 2$-point improvement from baseline compared with those receiving placebo (5.8\%; $p<0.001$ and $p<0.001$, respectively) [41]. Additionally, by week 12, a higher proportion of subjects receiving $200 \mathrm{mg}$ of drug and $100 \mathrm{mg}$ of drug (64.6\% and $40.7 \%$ ) obtained an EASI-75 compared with placebo (15.4\%; $p<0.001$ and $p=0.004$, respectively) [41]. Four serious TEAEs were reported in the treatment groups, including one case of pneumonia, one case of eczema herpeticum, and two cases of recurrence of herpes simplex. Gastrointestinal upset was also seen with slightly increased frequency in the group(s) receiving abrocitinib. Dose-dependent thrombocytopenia was also noted for doses $>10 \mathrm{mg}$, but this reversed by week 12 of treatment.

\subsection{Upadacitinib}

Upadacitinib is an oral small-molecule selective inhibitor of JAK1 [43]. Several phase III clinical trials evaluating upadacitinib in subjects with AD are ongoing (Table 2). In a phase IIb RCT in adults with $\mathrm{AD}$, by week 16 , a higher percentage of subjects receiving upadacitinib $(7.5,15$, or $30 \mathrm{mg}$ ) achieved an EASI-75 than the group receiving placebo ( $p \leq 0.001, p \leq 0.001, p \leq 0.05$, respectively) [44]. Additionally, by week 16, a higher proportion of subjects receiving upadacitinib $(7.5,15$, or $30 \mathrm{mg}$ ) achieved an IGA of 1 or 0 than the group receiving placebo $(p \leq 0.001$, 
$p \leq 0.001, p \leq 0.05$, respectively). Of note, this study is the first to evaluate selective JAK1 inhibition in AD patients without concomitant corticosteroid use. Only two serious TEAEs were reported in the treatment group, including jaw pericoronitis in a subject with a history of dental infections and worsening $\mathrm{AD}$ in another subject [44]. There were no dose-dependent adverse events. In a phase III RCT comparing upadacitinib and adalimumab in patients with severe rheumatoid arthritis, upadacitinib was generally well tolerated. However, the incidence of herpes zoster infection and elevations in CPK was higher in the group receiving upadacitinib [43].

\section{Conclusions}

Until recently, the treatment of moderate-to-severe AD relied on potent corticosteroids and systemic immunosuppressants, which can produce significant undesirable side effects. As moderate-to-severe AD can lead to poor quality of life, the development of targeted, well-tolerated immunomodulators remains important. An improved understanding of AD pathophysiology resulted in an explosion of research into new agents for this patient population. While the novel agents discussed here have demonstrated efficacy, others such as tezepelumab, apremilast, ustekinumab, and tradipitant failed to reach their primary endpoint in clinical trials $[17,28,45,46]$.

As new agents come to market, the tradeoff between efficacy and safety will be important. While the JAK inhibitors are effective in clinical trials and offer a much desired oral form of delivery, they are associated with a risk of serious adverse effects [47, 48]. There is an FDA mandated black box warning for risk of severe infection and death when using baricitinib $2 \mathrm{mg}$ in patients with rheumatoid arthritis [48]. This potential for serious adverse events is not surprising, as JAK inhibitors participate in signaling cascades that regulate both the acute inflammatory reaction and hematopoiesis [49, 50].

Leveraging the inhibition of specific subtypes of JAK proteins-only using selective JAK1 inhibitors (upadacitinib) in AD-may help minimize undesired side effects [50]. However, the higher dosages likely required for the treatment of autoimmune disease may overcome the selectivity of these agents at lower dosages [50]. Agents with a wide scope of action may carry a greater risk of serious adverse events compared with agents with a narrow scope of action, such as single interleukin inhibitors $[4,50]$. Currently, dupilumab is the only immunomodulator approved in the United States for moderate-to-severe AD, but this may change as several novel agents are successful in clinical trials. It may appear from dupilumab's performance in clinical trials that it insufficiently treats a large sub-group of patients with moderate-to-severe AD. However, the clinical landscape that these novel agents are entering may be different than anticipated, as dupilumab's meaningful clinical performance may be higher than might be expected [17]. Regardless, the benefit of developing several immunomodulators targeting distinct immune pathways is an increased probability of achieving disease control in all AD patients. Additional novel therapies are currently under investigation in clinical trials (APD334, KY1005, bermekimab, and many others). Future research will determine how these novel agents compare directly and if specific immunomodulators work better for certain subtypes of AD patients.

Author Contributions Literature search: MN, AMB. Drafting of the manuscript: $\mathrm{MN}, \mathrm{AMB}, \mathrm{EAB}$. Critical revision of the manuscript for important intellectual content: All authors. Supervision: SRF, LCS.

\section{Compliance with ethical standards}

Funding sources None.

Conflicts of interest Lindsay Strowd has received grants or support from Galderma, Pfizer, Actelion, and Sanofi Regeneron. Steven Feldman has received research, speaking, and/or consulting support from a variety of companies including Galderma, GSK/Stiefel, Almirall, Leo Pharma, Boehringer Ingelheim, Mylan, Celgene, Pfizer, Valeant, Abbvie, Samsung, Janssen, Lilly, Menlo, Merck, Novartis, Regeneron, Sanofi, Novan, Qurient, National Biological Corporation, Caremark, Advance Medical, Sun Pharma, Suncare Research, Informa, UpToDate, and the National Psoriasis Foundation. He is founder and majority owner of www.DrScore.com and founder and part owner of Causa Research, a company dedicated to enhancing patients' adherence to treatment. Megan Newsom, Arjun Bashyam, and Esther Balogh have no conflicts to disclose.

\section{References}

1. Furue K, Ito T, Tsuji G, Ulzii D, Vu YH, Kido-Nakahara M, et al. The IL-13-OVOL1-FLG axis in atopic dermatitis. Immunology. 2019;158(4):281-6. https://doi.org/10.1111/imm.13120.

2. Suarez-Farinas M, Ungar B, da Rosa CJ, Ewald DA, Rozenblit $\mathrm{M}$, Gonzalez J, et al. RNA sequencing atopic dermatitis transcriptome profiling provides insights into novel disease mechanisms with potential therapeutic implications. J Allergy Clin Immunol. 2015;135(5):1218-27. https://doi.org/10.1016/j.jaci.2015.03.003.

3. Czarnowicki T, He H, Krueger JG, Guttman-Yassky E. Atopic dermatitis endotypes and implications for targeted therapeutics. J Allergy Clin Immunol. 2019;143(1):1-11. https://doi. org/10.1016/j.jaci.2018.10.032.

4. Silverberg JI, Kantor R. The role of interleukins 4 and/or 13 in the pathophysiology and treatment of atopic dermatitis. Dermatol Clin. 2017;35(3):327-34. https://doi.org/10.1016/j. det.2017.02.005.

5. Bissonnette R, Pavel AB, Diaz A, Werth JL, Zang C, Vranic I, et al. Crisaborole and atopic dermatitis skin biomarkers: an intrapatient randomized trial. J Allergy Clin Immunol. 2019;144(5):1274-89. https://doi.org/10.1016/j.jaci.2019.06.047.

6. Tsoi LC, Rodriguez E, Degenhardt F, Baurecht H, Wehkamp U, Volks N, et al. Atopic dermatitis is an IL-13-dominant disease 
with greater molecular heterogeneity compared to psoriasis. J Invest Dermatol. 2019;139(7):1480-9. https://doi.org/10.1016/j. jid.2018.12.018.

7. Guttman-Yassky E, Krueger JG. Atopic dermatitis and psoriasis: two different immune diseases or one spectrum? Curr Opin Immunol. 2017;48:68-73. https://doi.org/10.1016/j.coi.2017.08.008.

8. Cabanillas B, Brehler A-C, Novak N. Atopic dermatitis phenotypes and the need for personalized medicine. Curr Opin Allergy Clin Immunol. 2017;17(4):309-15. https://doi.org/10.1097/ ACI.0000000000000376.

9. Weidinger S, Novak N. Atopic dermatitis. Lancet. 2016;387(10023):1109-22. https://doi.org/10.1016/S0140 $-6736(15) 00149-X$.

10. Guttman-Yassky E, Krueger JG, Lebwohl MG. Systemic immune mechanisms in atopic dermatitis and psoriasis with implications for treatment. Exp Dermatol. 2018;27(4):409-17. https://doi. org/10.1111/exd.13336.

11. Harb H, Chatila TA. Mechanisms of dupilumab. Clin Exp Allergy. 2019;50(1):5-14. https://doi.org/10.1111/cea.13491.

12. Wollenberg A, Howell MD, Guttman-Yassky E, Silverberg JI, Kell C, Ranade K, et al. Treatment of atopic dermatitis with tralokinumab, an anti-IL-13 mAb. J Allergy Clin Immunol. 2019;143(1):135-41. https://doi.org/10.1016/j.jaci.2018.05.029.

13. Simpson EL, Bieber T, Guttman-Yassky E, Beck LA, Blauvelt A, Cork MJ, et al. Two phase 3 trials of dupilumab versus placebo in atopic dermatitis. N Engl J Med. 2016;375(24):2335-48. https:// doi.org/10.1056/NEJMoa1610020.

14. de Bruin-Weller M, Thaci D, Smith CH, Reich K, Cork MJ, Radin A, et al. Dupilumab with concomitant topical corticosteroid treatment in adults with atopic dermatitis with an inadequate response or intolerance to ciclosporin A or when this treatment is medically inadvisable: a placebo-controlled, randomized phase III clinical trial (LIBERTY AD CAFE). Br J Dermatol. 2018;178(5):1083101. https://doi.org/10.1111/bjd.16156.

15. Deleuran M, Thaci D, Beck LA, de Bruin-Weller M, Blauvelt A, Forman S, et al. Dupilumab shows long-term safety and efficacy in moderate-to-severe atopic dermatitis patients enrolled in a phase 3 open-label extension study. J Am Acad Dermatol. 2019;82(2):377-88. https://doi.org/10.1016/j.jaad.2019.07.074.

16. Simpson EL, Paller AS, Siegfried EC, Boguniewicz M, Sher L, Gooderham MJ, et al. Efficacy and safety of dupilumab in adolescents with uncontrolled moderate to severe atopic dermatitis: a phase 3 Randomized Clinical Trial. JAMA Dermatol. 2019. https ://doi.org/10.1001/jamadermatol.2019.3336.

17. Wang C, Kraus CN, Patel KG, Ganesan AK, Grando SA. Realworld experience of dupilumab treatment for atopic dermatitis in adults: a retrospective analysis of patients' records. Int J Dermatol. 2020;59(2):253-6. https://doi.org/10.1111/ijd.14573.

18. Nahum Y, Mimouni M, Livny E, Bahar I, Hodak E, Leshem YA. Dupilumab-induced ocular surface disease (DIOSD) in patients with atopic dermatitis: clinical presentation, risk factors for development and outcomes of treatment with tacrolimus ointment. $\mathrm{Br}$ J Ophthalmol. 2019. https://doi.org/10.1136/bjophthalmol-2019315010.

19. Uchida H, Kamata M, Nagata M, Fukaya S, Hayashi K, Fukuyasu A, et al. Conjunctivitis in patients with atopic dermatitis treated with dupilumab is associated with higher baseline serum levels of immunoglobulin $\mathrm{E}$ and thymus and activation-regulated chemokine but not clinical severity in a real-world setting. J Am Acad Dermatol. 2019. https://doi.org/10.1016/j.jaad.2019.12.039.

20. Cork MJ, Thaci D, Eichenfield LF, Arkwright PD, Hultsch T, Davis JD, et al. Dupilumab in adolescents with uncontrolled moderate-to-severe atopic dermatitis: results from a phase IIa openlabel trial and subsequent phase III open-label extension. Br J Dermatol. 2019;182(1):85-96. https://doi.org/10.1111/bjd.18476.
21. Akinlade B, Guttman-Yassky E, de Bruin-Weller M, Simpson EL, Blauvelt A, Cork MJ, et al. Conjunctivitis in dupilumab clinical trials. Br J Dermatol. 2019;181(3):459-73. https://doi. org/10.1111/bjd.17869.

22. Barroso-García B, Rial MJ, Molina A, Sastre J. Alopecia areata in severe atopic dermatitis treated with dupilumab. J Investig Allergol Clin Immunol. 2018;28(6):420-1. https://doi.org/10.18176/ jiaci.0301.

23. Flanagan K, Sperling L, Lin J. Drug-induced alopecia after dupilumab therapy. JAAD Case Rep. 2018;5(1):54-6. https://doi. org/10.1016/j.jdcr.2018.10.010.

24. Mitchell K, Levitt J. Alopecia areata after dupilumab for atopic dermatitis. JAAD Case Rep. 2018;4(2):143-4. https://doi. org/10.1016/j.jdcr.2017.11.020.

25. Mohan GC, Silverberg JI. Association of vitiligo and alopecia areata with atopic dermatitis: a systematic review and metaanalysis. JAMA Dermatol. 2015;151(5):522-8. https://doi. org/10.1001/jamadermatol.2014.3324.

26. Paller AS, Bansal A, Simpson EL, Boguniewicz M, Blauvelt A, Siegfried EC, et al. Clinically meaningful responses to dupilumab in adolescents with uncontrolled moderate-to-severe atopic dermatitis: post-hoc analyses from a Randomized Clinical Trial. Am J Clin Dermatol. 2020;21(1):119-31. https://doi. org/10.1007/s40257-019-00478-y.

27. Silverberg JI, Simpson EL, Ardeleanu M, Thaci D, Barbarot S, Bagel J, et al. Dupilumab provides important clinical benefits to patients with atopic dermatitis who do not achieve clear or almost clear skin according to the Investigator's Global Assessment: a pooled analysis of data from two phase III trials. Br J Dermatol. 2019;181(1):80-7. https://doi.org/10.1111/bjd.17791

28. Uchida H, Kamata M, Mizukawa I, Watanabe A, Agematsu A, Nagata M, et al. Real-world effectiveness and safety of dupilumab for the treatment of atopic dermatitis in Japanese patients: a single-centre retrospective study. Br J Dermatol. 2019;181(5):1083-5. https://doi.org/10.1111/bjd.18163.

29. Ruiz-Villaverde R, Dominguez-Cruz J, Armario-Hita JC, Martinez-Pilar L, Alcantara-Luna S, Pereyra-Rodriguez JJ. Dupilumab: short-term effectiveness and security in real clinical practice - A retrospective multicentric study. J Eur Acad Dermatol Venereol. 2019;33(1):e21-2. https://doi.org/10.1111/ jdv.15118.

30. LEO Pharma announces positive top-line results for tralokinumab from three Phase 3 studies in adult patients with moderate-to-severe AD. 2019. https://via.ritzau.dk/pressemeddelelse/ leo-pharma-announces-positive-top-line-results-for-tralokinum ab-from-three-phase-3-studies-in-adult-patients-with-moderateto-severe-ad?publisherId $=12353927 \&$ release $I d=13584831$. Accessed 2 Apr 2020.

31. Baverel P, She D, Piper E, Ueda S, Yoshioka T, Faggioni R, et al. A randomized, placebo-controlled, single ascendingdose study to assess the safety, tolerability, pharmacokinetics, and immunogenicity of subcutaneous tralokinumab in Japanese healthy volunteers. Drug Metab Pharmacokinet. 2018;33(3):150-8. https://doi.org/10.1016/j.dmpk.2017.12.001.

32. Simpson EL, Flohr C, Eichenfield LF, Bieber T, Sofen H, Taieb A, et al. Efficacy and safety of lebrikizumab (an antiIL-13 monoclonal antibody) in adults with moderate-to-severe atopic dermatitis inadequately controlled by topical corticosteroids: A randomized, placebo-controlled phase II trial (TREBLE). J Am Acad Dermatol. 2018;78(5):863-71e11. https://doi. org/10.1016/j.jaad.2018.01.017.

33. Guttman-Yassky E, Blauvelt A, Eichenfield LF, Paller AS, Armstrong AW, Drew J, et al. Efficacy and safety of lebrikizumab, a high-affinity interleukin 13 inhibitor, in adults with moderate to severe atopic dermatitis: a phase $2 \mathrm{~b}$ Randomized Clinical 
Trial. JAMA Dermatol. 2020. https://doi.org/10.1001/jamad ermatol.2020.0079.

34. Ruzicka T, Hanifin JM, Furue M, Pulka G, Mlynarczyk I, Wollenberg A, et al. Anti-interleukin-31 receptor a antibody for atopic dermatitis. N Engl J Med. 2017;376(9):826-35. https:// doi.org/10.1056/NEJMoa1606490.

35. Silverberg JI, Pinter A, Pulka G, Poulin Y, Bouaziz JD, Wollenberg A, et al. Phase 2B randomized study of nemolizumab in adults with moderate-to-severe atopic dermatitis and severe pruritus. J Allergy Clin Immunol. 2019;145(1):173-82. https:// doi.org/10.1016/j.jaci.2019.08.013.

36. Nemoto O, Furue M, Nakagawa H, Shiramoto M, Hanada R, Matsuki S, et al. The first trial of CIM331, a humanized antihuman interleukin-31 receptor A antibody, in healthy volunteers and patients with atopic dermatitis to evaluate safety, tolerability and pharmacokinetics of a single dose in a randomized, double-blind, placebo-controlled study. Br J Dermatol. 2016;174(2):296-304. https://doi.org/10.1111/bjd.14207.

37. Kabashima K, Furue M, Hanifin JM, Pulka G, Wollenberg A, Galus R, et al. Nemolizumab in patients with moderate-tosevere atopic dermatitis: randomized, phase II, long-term extension study. J Allergy Clin Immunol. 2018;142(4):1121-30. https ://doi.org/10.1016/j.jaci.2018.03.018.

38. Guttman-Yassky E, Pavel AB, Zhou L, Estrada YD, Zhang N, $\mathrm{Xu} \mathrm{H}$, et al. GBR 830, an anti-OX40, improves skin gene signatures and clinical scores in patients with atopic dermatitis. J Allergy Clin Immunol. 2019;144(2):482-93e7. https://doi. org/10.1016/j.jaci.2018.11.053.

39. Guttman-Yassky E, Silverberg JI, Nemoto O, Forman SB, Wilke A, Prescilla $R$, et al. Baricitinib in adult patients with moderateto-severe atopic dermatitis: a phase 2 parallel, double-blinded, randomized placebo-controlled multiple-dose study. J Am Acad Dermatol. 2019;80(4):913-21e9. https://doi.org/10.1016/j. jaad.2018.01.018.

40. Simpson EL, Lacour JP, Spelman L, Galimberti R, Eichenfield $\mathrm{LF}$, Bissonnette R, et al. Baricitinib in patients with moderateto-severe atopic dermatitis and inadequate response to topical corticosteroids: results from two randomized monotherapy phase III trials. Br J Dermatol. 2020. https://doi.org/10.1111/ bjd. 18898 .

41. Gooderham MJ, Forman SB, Bissonnette R, Beebe JS, Zhang W, Banfield C, et al. Efficacy and safety of oral janus kinase 1 inhibitor abrocitinib for patients with atopic dermatitis: a phase 2 randomized clinical trial. JAMA Dermatol. 2019. https://doi. org/10.1001/jamadermatol.2019.2855.

42. Pfizer Announces Positive Top-line Results From Phase 3 Study of Investigational Oral Jak1 Candidate, Abrocitinib
(Pf-04965842), in Patients Aged 12 and Older With Moderate to Severe Atopic Dermatitis. 2019. https://www.pfizer.com/ news/press-release/press-release-detail/pfizer_announces_posit ive_top_line_results_from_phase_3_study_of_investigational _oral_jak1_candidate_abrocitinib_pf_04965842_in_patients_ aged_12_and_older_with_moderate_to_severe_atopic_derma titis. Accessed 10 Apr 2020.

43. Fleischmann R, Pangan AL, Song IH, Mysler E, Bessette L, Peterfy C, et al. Upadacitinib versus placebo or adalimumab in patients with rheumatoid arthritis and an inadequate response to methotrexate: results of a phase III, double-blind. Randomized Controlled Trial. Arthritis Rheumatol. 2019;71(11):1788-800. https://doi.org/10.1002/art.41032.

44. Guttman-Yassky E, Thaçi D, Pangan AL, Hong HC-H, Papp KA, Reich K, et al. Upadacitinib in adults with moderate to severe atopic dermatitis: 16-week results from a randomized, placebocontrolled trial. J Allergy Clin Immunol. 2020;145(3):877-84. https://doi.org/10.1016/j.jaci.2019.11.025.

45. Agnihotri G, Lio PA. revisiting therapies for atopic dermatitis that failed clinical trials. Clin Drug Investig. 2020. https://doi. org/10.1007/s40261-020-00905-7.

46. Park B. Tradipitant Misses Primary End Point in Atopic Dermatitis Trial. 2020. https://www.empr.com/home/news/tradi pitant-misses-primary-end-point-in-atopic-dermatitis-trial/. Accessed 8 Apr 2020.

47. Lilly Announces Top-Line Phase 3 Results for Baricitinib in Patients with Moderate to Severe Atopic Dermatitis. 2019. https://investor.lilly.com/news-releases/news-release-details/ lilly-announces-top-line-phase-3-results-baricitinib-patients. Accessed 8 Apr 2020.

48. Napolitano M, Fabbrocini G, Cinelli E, Stingeni L, Patruno C. Profile of baricitinib and its potential in the treatment of moderate to severe atopic dermatitis: a short review on the emerging clinical evidence. J Asthma Allergy. 2020;13:89-94. https://doi. org/10.2147/jaa.S206387.

49. Babon JJ, Kershaw NJ, Murphy JM, Varghese LN, Laktyushin A, Young SN, et al. Suppression of cytokine signaling by SOCS3: characterization of the mode of inhibition and the basis of its specificity. Immunity. 2012;36(2):239-50. https:// doi.org/10.1016/j.immuni.2011.12.015.

50. Schwartz DM, Kanno Y, Villarino A, Ward M, Gadina M, O'Shea JJ. JAK inhibition as a therapeutic strategy for immune and inflammatory diseases. Nat Rev Drug Discov. 2017;16(12):84362. https://doi.org/10.1038/nrd.2017.201. 